OAI-PMH: http://www.indteca.com/ojs/index.php/Revista Scientific/oai

Ensayo Original / Original Essay

\title{
La Cibercultura como Herramienta para Edificar Mundos Contemporáneos, Reales y Virtuales
}

\author{
Autora: Merry Carmen Domínguez Moreno \\ Universidad Nacional Experimental "Rafael María Baralt", UNERMB \\ merry dominguez@hotmail.com \\ Trujillo, Venezuela
}

\section{Resumen}

El propósito de este ensayo luego del análisis de las ideas formuladas por diferentes autores mencionados a lo largo del texto, es mostrar a la comunidad científica e interesados en el tema, analizar su inquietud respecto a la Cibercultura como herramienta para edificar mundos contemporáneos, reales y virtuales. Se trató el procesamiento de la información con miras a sustentar el análisis de los esbozos teóricos pertenecidos con la Cibercultura como una forma de compartir conocimientos, a partir de un mundo contemporáneo real y virtual para modificar las formas de inquietar, proceder y conocer en la realidad social que trasladen a la articulación curricular. Se concluye que, las aplicaciones teóricas deben ser analizadas desde las prácticas pedagógicas con la idea de revalorizar el uso y aprovechamiento de las herramientas tecnológicas en el proceso de enseñanza-aprendizaje, en la búsqueda de optimar la calidad de formación en todos los niveles y modalidades del sistema educativo venezolano.

Palabras clave: enseñanza multimedia; arte digital; educación. 


\title{
The Cyberculture as a tool to build contemporary, real and virtual worlds
}

\begin{abstract}
The purpose of this essay after the analysis of the ideas formulated by different authors mentioned throughout the text, is to show the scientific community and interested in the subject, analyze their concern about the Cyberculture as a tool to build contemporary worlds, real and virtual The processing of the information was treated with a view to supporting the analysis of the theoretical sketches belonging to the Cyberculture as a way of sharing knowledge, from a real and virtual contemporary world to modify the ways of disturbing, proceeding and knowing in reality social transfer to the curricular articulation. It is concluded that the theoretical applications must be analyzed from the pedagogical practices with the idea of revaluing the use and use of technological tools in the teaching-learning process, in the search of optimizing the quality of training at all levels and modalities of the Venezuelan educational system.
\end{abstract}

Keywords: multimedia instruction; digital art; education.

Date Received: 08-05-2018

Date Acceptance: 25-07-2018 


\section{Introducción}

El estudio mostrado lleva por título La Cibercultura como Herramienta para Edificar Mundos Contemporáneos, Reales y Virtuales, el cual se corresponde con un ensayo donde se plasmarán características de esta herramienta virtual, a favor de utilizarla para edificar mundos en esta era posmoderna adaptada a la realidad circundante. Además, darle realce a los espacios de tecnología que hoy invaden para satisfacción e información y conocimiento nuestros entornos universitarios.

En la apertura del siglo XXI, las universidades desafían un proceso de estudio y cambio de sus estructuras, en el cual, existe afectación de las prioridades sociales en vías de globalizarse, provocando la ciencia y la tecnología al servicio del desarrollo de la educación universitaria como agente de cambio en una cultura emergente.

En cualquier caso, a nivel mundial y nacional una de las inquietudes por parte de las Instituciones de Educación Universitaria (IEU) es lograr alinear la formación del personal universitario hacia la incorporación de modalidades de estudios virtuales a través de eventos que discurran respecto de las competencias de los actores implicados en el asunto de enseñanza. Evidentemente, con relación a la Educación Virtual, según Rubio y Romero (2005): establecen que "las (IEU) deben interesarse en la creación de programas más flexibles que brinden la posibilidad de relacionarse con el alumno fuera del aula de clases" (pág. 2).

En otras palabras, el tipo de aprendizaje virtual debe ser modificado por las instituciones que les competa la educación universitaria, tomando en cuenta ciertas debilidades que aún prevalecen en los actuales currículos para que los estudiantes sean capaces de adaptarse a los diferentes contextos virtuales que hoy ofrecen las tecnologías del aprendizaje. Tal como lo señala, Martínez (2016):

Estos cambios vertiginosos de la información están causando 
enormes transformaciones en todos los campos de nuestra sociedad y la educación no es una excepción. Esas tecnologías se presentan cada vez más, como una necesidad en el contexto de sociedad, donde los rápidos cambios, el aumento de los conocimientos y las demandas de una educación de alto nivel, constantemente actualizada, se convierten en una exigencia permanente (pág. 3).

De cualquier manera, todo cambio debe ser aceptado como herramienta para transformar las realidades que subyacen en el mundo tecnológico, no olvidando que educación y tecnología en estos tiempos globalizados, van de la mano para ser insertadas en contextos sociales y culturales. En consecuencia, dada la importancia de todo lo señalado por la autora, el presente ensayo busca analizar la Cibercultura como herramienta para edificar mundos contemporáneos, reales y virtuales. Tal como lo describe Escobar (2005):

Tanto en las comunidades técnico-científicas, como en las ciencias sociales, han comenzado a conformarse nuevas perspectivas. En estas últimas, un campo completo de enseñanza e investigación alrededor de dos proyectos distintos pero relacionados: (a) los estudios de la ciencia y la tecnología y (b) los programas de ciencia, tecnología y sociedad (pág. 16).

Parafraseando al autor, se concibe un camino en la indagación científica que admite el estudio de la tecnología, no sólo, desde la génesis y evolución de ésta, sino también de las contradicciones en todas las áreas y niveles donde dicha tecnología se inserta y desarrolla. Por esto, al hablar del vocablo Cibercultura, a juicio de Lévy (1999a), citado en Salas (2013): se entiende como “...el conjunto de técnicas, de maneras de hacer, de maneras de ser, de valores, de representaciones que están relacionadas con la extensión del Ciberespacio..." (pág. 2).

Por ende, el camino recorrido para comunicarse unos con otros, significa para muchos y en el mío en particular parte de mi vida cotidiana. 
Aunado, a otras terminologías tecnológicas existentes, como televisión por cable o usanza de aparatos de inscripción tecnológica como celulares, computadoras, cajeros automáticos, otros.

No obstante, las comunidades científicas a lo largo del cosmos se han dedicado a estudiar de qué forma estos progresos tecnológicos impactan en la cultura y socialización del individuo. Por esto, la autora del ensayo mostrado, como resultado de una revisión analítica y crítica de la información objeto de estudio, busca analizar su inquietud respecto a la Cibercultura como herramienta si es útil para ¿edificar mundos contemporáneos, reales y virtuales?

\section{Análisis de Bosquejos Teóricos}

Mirando nuestro alrededor, se observa en este momento una sociedad diferente donde se le sigue dando al texto su lugar como efigie que exige, decide, instituye, sin embargo, tristemente la lectura se desempeñó, es decir otro mundo se edificó, aunado a posibilidades de interpretar, dar significado a nuevos términos que hasta hace poco eran desconocidos. Me refiero, a la relevancia de la Cibercultura, enfocada por mi criterio como una herramienta tácita, que aplicada de manera responsable y objetiva pueda servir en estos tiempos de la posmodernidad para afrontar realidades en el campo virtual. Es así, como el espectáculo de la cultura es la multiplicidad, en las que los seres humanos han sistematizado el sentido de sus vidas. La cultura, a juicio de Cáceres (1998):

Es el espectáculo de lo humano por excelencia ...Esa es la primera impresión, como algo calificable de humano puede tomar tan variadas formas; es asombroso. Y si la mirada se dirige hacia el pasado el efecto es tan o más cautivador... (pág. 13).

En otras palabras, la cultura ha permanecido en nuestra cotidianidad 
muchas veces ocultando las formas culturales, donde echar un vistazo a lo cultural es mirar, dar una ojeada, observar mediante los sentidos, configurar los significados de lo que realmente significa esa cultura. De ahí, la importancia de la Cibercultura, porque en este momento ciertas sociedades de la información se abrieron para cambiar, o cambiaron para abrirse a nuevas posibilidades, lo que es lo mismo, sus intereses de estar informados crecieron al ver que no era posible resolver situaciones presentes en la sociedad, y su implementación en el campo cultural es cada vez más significativo a favor de las comunidades y entornos.

Es de esperar entonces, encauzar el hilo conductor de la discusión en torno a la preponderancia del ciberespacio, y su influencia en la vida cotidiana socio cultural de los seres humanos, hasta conseguir analizar la analogía de este espacio virtual, como aspecto fundamental del desarrollo social: la educación y en particular la educación universitaria. De este modo, una de las herramientas más demostrativa de la llegada de la Cibercultura a los entornos educativos es el hipertexto.

Sin embargo, una definición más acorde fue la presentada por Caridad y Moscoso (1991): quien precisa el hipertexto como "la creación y representación de enlaces entre distintas partes de información que son los nudos, cualquier palabra, frase, párrafo puede ser según, esta idea, un nudo, si aparece asociado, mediante un enlace, con otras palabras, frases 0 párrafos" (págs. 38-40).

Interpretando los supuestos, esta herramienta de texto brinda diferentes oportunidades porque se lee el documento de forma lineal o en secuencia si es del agrado del lector o bien, puede efectuar lectura navegando disfrutando de los múltiples vincules que el hipertexto ofrece. Esta modalidad, es de relevancia con nuestros estudiantes, siempre y cuando el facilitador, esté capacitado debidamente en la forma cómo se debe realizar para que pueda surtir efectos positivos tanto en los estudiantes como en los procesos 
pedagógicos que éstos puedan implementar.

No obstante, al fijar posición sobre la implementación de los hipertextos y sistemas hipertextuales, se nota que actualmente dentro de la Cibercultura y por ende en los ambientes educativos se debería analizar su uso en los programas educativos como herramienta pedagógica, aunque se deban tomar en cuenta ciertos aspectos para ser analizados.

Asumiendo una postura ante tales afirmaciones, lo actual es que los hipertextos y los medios hipertextuales dentro de la Cibercultura y por ende en los ambientes educacionales deben sentirse comprometidos en analizar su usanza dentro de las tecnologías pedagógicas, asumiendo algunas consideraciones en sus observaciones. Que pudieran ser el tipo de población estudiantil que atiende, los espacios tecnológicos que funcionen en la institución, además de la preparación suficiente que posea el docente en esta modalidad virtual. Asimismo, Rodríguez (2007), expresa:

los mundos digitales no son como los mundos de la naturaleza: son mundos artificiales hechos por hombres a través de computadores. Como en los artefactos del arte tradicional (pintura, teatro, música, literatura, poesía y escultura), todo allí es imaginación; pero los mundos digitales tienen el potencial para poner en marcha ideas y profundas emociones de un modo que otros medios no podrían hacerlo. Mundos que en todo caso no podrían ser expresados sin la tecnología digital (pág. 5).

La posición del autor, relacionada con los mundos digitales en relación a la Cibercultura es un aspecto, que como perfil de los futuros egresados, reúne o engloba todo lo que hemos analizado hasta aquí, y se refiere a la iniciativa que se deben revisar los modelos de aprendizaje que se demandan para enseñar a través de los mundos digitales. Donde los sujetos posean características comunes, concebidas como lo inmerso en todo lo tecnológico, quienes navegan en la Internet con excesiva fluidez y conocimientos digitales.

Al mismo tiempo, vivimos en un mundo donde las tecnologías de la 
información y comunicación tienen figuración como no la habían alcanzado antecedentemente en ninguna época de desarrollo de la humanidad. De manera contundente, no podemos negar, que Internet ha establecido una red global para que se enlacen los sujetos, y puedan comunicarse entre ellos y para que puedan trabajar en la virtualidad. De hecho, no estaría mal reconocer desde el principio según Cabero y Gisbert (2005):

La idea de comunidad ha sido una de las bases de configuración de Internet desde sus comienzos ...no podemos olvidarnos que desde sus comienzos los científicos e investigadores la han utilizado para compartir datos, realizar de forma conjunta investigaciones y proyectos... (pág. 6).

Por esto, al ofrecer una definición de Comunidad Virtual, encontramos un doble problema, primeramente, la propia enunciación del término comunidad, y por otra con la referencia del término virtual. Respecto al término comunidad, lo señala Baym (2002): es muy complejo de definir, "ya que tiene connotaciones descriptivas, normativas e ideológicas y abarca tanto dimensiones materiales como simbólicas" (pág. 55). Es decir, al conceptualizarlo no puede hacerse unívocamente hay que enfocarlo desde diferentes configuraciones, que van desde la ideológica a la experta y técnica, sin olvidarnos de sus relaciones religiosas.

Entretanto, lo que respecta a lo virtual suele inscribirse con connotaciones de secundario, no real poco significativo. Así lo expone Lévy (1999b): "en su uso corriente, el término virtual, se suele emplear para expresar la ausencia pura y simple de existencia, presuponiendo la realidad como una realización material, y una presencia tangible" (pág. 17). Interpretando los supuestos, lo real estaría en el orden del yo como persona y lo virtual a mi forma de entender también puede ser real porque hay sujetos de por medio como el caso que nos compete de la Cibercultura.

Dentro de este orden de ideas, la educación virtual se ha creado de las 
modalidades educativas con asiento en la distancia. Su aparición, se genera a partir de los cambios y demandas sociales, en las que prohibiciones económicas, materiales, espaciales, no fueron tomadas en cuenta por la educación presencial, la virtualidad no tiene límites, reglas, es espontánea y transformadora puesto que parte de las virtudes humanas. Por esto, se hace necesario definir de acuerdo con la interpretación que hace Barberá (2001):

El entorno virtual hace referencia a un contexto tecnológico, que a su vez, está relacionado con otros argumentos. Como plataforma tecnológica reseña un aspecto mucho más rico y complejo, es un nivel micro reservado para el propio proceso de aprendizaje (pág. 23).

De este modo, se entiende entonces que la Cibercultura tratada en el presente ensayo como herramienta para edificar mundos contemporáneos, reales y virtuales, se caracteriza porque permitirá crear un marco de participación didáctica entre el profesor y los estudiantes. Su importancia radica entonces en la manera en que la universidad al incorporar dentro de sus políticas el desarrollo de proyectos reales y virtuales instituye una circunstancia globalizadora de la educación a través del camino electrónico y el alcance de la información por este medio. Además, servirá para propiciar el pensamiento favorable, creativo ofreciendo al docente la oportunidad de trabajar en cooperación, con sentido colaborativo.

\section{Síntesis del Tópico Objeto de Revisión}

El sistema educacional en general, enfrenta retos y desafíos ante la nueva forma de enseñanza a través de tecnicismos, porque permite gestionar la capacitación, para transformar la información en conocimiento, hacer de éste un elemento de transformación social, cultural que garantice, el progreso del entorno para uso responsable de redes sociales virtuales.

Comprender que estos retos y desafíos están abocados a 
emprendimientos investigativos, capaces de diseñar herramientas que permitan edificar mundos contemporáneos reales y virtuales, en este caso con la finalidad de cultivar las bondades de la Cibercultura para el fortalecimiento de los procesos educativos.

Por ende, en educación, La Cibercultura llegó para permanecer y no tiene por qué desplazar ni agrupar las culturas de los pueblos, más bien, los procesos educativos deben ser los integradores de culturas de manera tal, que sirvan para rescatar óptimo de cada una para permitir el desarrollo íntegro de las sociedades y sus constituyentes.

En este ensayo, se abordaron temáticas de la Cibercultura que fueron analizadas como bosquejos teóricos, considerados relevantes para conocer su utilidad en la sociedad de este mundo contemporáneo que desea se construyan espacios comunes que extiendan el potencial de la cibercultura en la educación.

De manera concluyente, es necesario considerar que la Cibercultura tiene grandes ventajas que ofrecer, en educación, en todos los niveles, y desde misceláneos ámbitos y organizaciones, no quedando anclada en el pasado. Más bien, debe proporcionar espacios que inviten a la reflexión e investigación dificultosa y profunda que permita superar los obstáculos de este nuevo mundo real y digital, capaz de promover la unificación y actualización en los procesos y modelos de enseñanza-aprendizaje que les permitan, a las comunidades digitales optimas alternativas para el desarrollo de las comunidades donde se desenvuelven.

\section{Referencias}

Barberá, E. (Coord.) (2001). La Incógnita de la Educación a Distancia. Cuadernos de Educación ICE. España: Universitat de Barcelona, Horsori Editorial.

Baym, N. (2002). Interpersonal Life Online. In S. Livingston \& L. Lievrouw 
(eds.). The Handbook of New Media. London: SAGE Publications Ltd. Cabero, J., \& Gisbert, M. (2005): La Formación en Internet. Guía para el Diseño de Materiales Formativos. Sevilla, España: MAD.

Cáceres, J. (1998). Cibercultura, Ciberciudad, Cibersociedad hacia la Construcción de Mundos Posibles en nuevas Metáforas Conceptuales. Estudios sobre las Culturas Contemporáneas, IV(7), 923. Recuperado de: http://www.redalyc.org/articulo.oa?id=31600702 Caridad, M., \& Moscoso, P. (1991). Los Sistemas de Hipertexto e Hipermedios: Una Nueva Aplicación en Informática Documental. Madrid, España: Fundación Germán Sánchez Ruipérez.

Escobar, A. (2005). Bienvenidos a la Cyberia. Notas para una Antropología de la Cibercultura. Revista de Estudios Sociales, (22), 15-35. Recuperado de:

https://revistas.uniandes.edu.co/doi/abs/10.7440/res22.2005.01

Lévy, P. (1999a,b). Ciberespacio y Cibercultura. Barcelona, España: Universitat Oberta de Catalunya.

Martínez, O. (2016). Programa de Formación Docente de las Tecnologías del Aprendizaje y el Conocimiento (TAC) En la Universidad Pedagógica Experimental Libertador Núcleo Barinas (Venezuela). Revista Scientific, 1(1), 90-114. Recuperado de:

https://doi.org/10.29394/scientific.issn.2542-2987.2016.1.1.6.90-114

Rodríguez, J. (2007). La crítica hoy: De los retos de la posmodernidad a los retos de la Cibercultura. Ponencia presentada en el Encuentro de crítica: perspectivas y problemas de la crítica literaria en el siglo XXI. Bogotá, Colombia: Universidad Nacional de Colombia. Recuperado de: http://cmap.javeriana.edu.co/servlet/SBReadResourceServlet?rid=126 442485159218989218821618

Rubio, M., \& Romero, L. (2005). Apostar por la Calidad de la Educación Superior a Distancia desde la Pertinencia Social. Ecuador: 
Universidad Técnica Particular de Loja.

Salas, Y. (2013). Cibercultura y Educación. Diá-logos (San Salvador), 7(11), 29-43, ISSN: 1996-1642. Recuperado de:

http://rd.udb.edu.sv:8080/jspui/bitstream/11715/454/1/2.\%20Cibercultu ra\%20y\%20educacion.pdf 
Merry Carmen Domínguez Moreno

e-mail: merry dominguez@hotmail.com

Nacida en Venezuela, estado Trujillo. Postgrado en

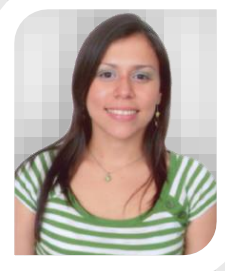

Docencia para la Educación Superior: Universidad Nacional Experimental "Rafael María Baralt" (Escolaridad Culminada en el 2010) en Valera, estado Trujillo. Ingeniero de Computación: egresada de la Universidad Valle del Momboy (2005) en Valera, Estado Trujillo. Coordinadora de la Unidad de Sistemas en el Instituto Universitario de Tecnología "Mario Briceño Iragorry" en Carora, Estado Lara. (2005-2006). Docente Contratada del Instituto Universitario de Tecnología del Estado Trujillo. (2010-2012). Docente Contratada. Universidad Politécnica Territorial del Estado Trujillo "Mario Briceño Iragorry". Desde el 2010 hasta la actualidad.

El contenido de este manuscrito se difunde bajo una Licencia de Creative Commons ReconocimientoNoComercial-Compartirlgual 4.0 Internacional 